\title{
Educación ambiental: una alternativa para la conservación del manglar
}

\author{
Reyna Marisol Linares Mazariegos ${ }^{1}$ \\ Cristian Tovilla Hernández ${ }^{2}$ \\ Juan Carlos De la Presa Pérez ${ }^{3}$
}

\begin{abstract}
RESUMEN
El manglar constituye un ecosistema irremplazable, del cual las comunidades humanas extraen diversos recursos para sobrevivir. Resulta fundamental divulgar la importancia de este ecosistema como refugio de muchas especies de la flora y la fauna así como el uso racional del mismo. La educación ambiental es necesaria para ayudar a la concientización sobre la problemática que enfrentan los bosques de manglares en la actualidad. Las actividades de Educación Ambiental constituyen parte del proyecto Restauración de áreas de manglar impactadas por obras de dragado en el sistema lagunar de Chantuto que desde abril de 2003 realiza El Colegio de la Frontera Sur con financiamiento de la Comisión Nacional de Acuacultura y Pesca (CONAPESCA) en las Escuelas Primarias Juan Escutia y Francisco Villa en la Comunidad de Santa Isabel, dentro de la Reserva de la Biosfera La Encrucijada. El objetivo es que mediante pláticas, exposición de videos, juegos y otras actividades escolares se cree conciencia en los niños de nivel primaria sobre la importancia ecológica y económica del cuidado y la preservación de los recursos del manglar en beneficio de las comunidades. Los temas principales son: el manglar y sus recursos; los principales impactos de las actividades sobre la zona costera; la importancia de la conservación, el manejo y la restauración de los bosques de manglar; las lagunas costeras y estuarios; la problemática de las obras de dragado sobre estos sistemas; el manejo del agua, el suelo, la basura y el cuidado de la salud, etc. Adicionalmente, mediante talleres para la protección del manglar, se ha logrado involucrar a algunas personas mayores en este proyecto, a través de actividades que muestran la problemática y acciones para un aprovechamiento racional del manglar y sus recursos.
\end{abstract}

PALABRAS CLAVE:

Conservación, educación ambiental, manejo, manglar.

\begin{abstract}
Mangroves constitutes an irreplaceable ecosystem, from which human communities extract diverse resources to survive. It is fundamental to inform the public about the importance of this ecosystem as a refuge of many species of the flora and fauna as well as its rational use. The environmental education is necessary to help in the awareness on the problematic that mangrove forests face at the present time. The activities of environmental education constitute part of the project Restauracion de areas de manglar impactadas por obras de dragado en el sistema lagunar de Chantuto initiated in April of 2003 by El Colegio de la Frontera Sur with funding from the Comision Nacional de Acuacultura y Pesca (CONAPESCA), at the Juan Escutia and Francisco Villa Elementary Schools in the Community of Santa Isabel, within the Reserva de la Biosfera La Encrucijada. The objective is that by using conferences, exhibition of videos, games and other activities, to create conscience in the children of elementary school level about the ecological and economic importance of the care and preservation of the resources of mangroves in benefit of the communities. The main subjects are: Mangrove and its resources, main impacts of the activities on the coastal zone, importance of the conservation, handling and restoration of the forests of mangrove, the coastal lagoons and estuaries, problematic of works of dredging on these systems, handling of the water, the soil, garbage and human well being, etc. Additionally by means of shops for the protection of mangrove it has been possible to involve senior citizens in this project, through activities that show the problematic and actions for a rational usage of mangrove and its resources.
\end{abstract}

KEY WORDS:

Conservation, environmental education, forest management, mangroves. km 2.5. Tapachula 30700 Chiapas. c.e.: rlinares@tap-ecosur.edu.mx, ctovilla@tap-ecosur.edu.mx. 


\section{INTRODUCCIÓN}

La costa de Chiapas comprende un amplio litoral de aproximadamente 310 kilómetros sobre el Océano Pacífico, cuenta con alrededor de 115828 hectáreas de esteros y lagunas costeras (RuizDurá, 1985), dentro de estos sobresalen grandes y complejos macizos forestales de manglar y amplias zonas pantanosas de tulares y popales, así como relictos de selvas medianas y bajas. Todos estos tipos de bosques existen dentro de la Reserva de la Biosfera La Encrucijada, una de las regiones de humedales más extensa, diversa y productiva de México.

Los manglares son formaciones vegetales de estructura densa y bien definida, son uno de los ecosistemas más productivos y el punto de partida de la cadena trófica; son además el resultado de la interacción entre el medio terrestre y el acuático (Tovilla, 1998). Los bosques de manglar de la Reserva de la Biosfera La Encrucijada están considerados como unos de los mejor desarrollados del Pacífico Americano. En esta área, los manglares están concentrados a lo largo de la franja costera alrededor del sistema lagunar Chantuto-Panzacola, con árboles de $20 \mathrm{~m}$ a $35 \mathrm{~m}$ de altura.

Son importantes como hábitat de una gran variedad de fauna, como refugio y sitio de anidación de aves residentes, albergan a especies amenazadas y en peligro de extinción, proporcionan las condiciones para el desarrollo de estadios juveniles de diversas especies marinas, permiten la formación de suelos y con ello la sucesión natural, además de tener un papel preponderante en las actividades socioeconómicas de los habitantes de la región (Tovilla, 1998).

Sin embargo, el hombre no siempre percibe el significado y la enorme importancia de este ecosistema; los manglares han sido degradados o destruidos por la extracción de productos forestales, tala parcial o total, ampliación de fronteras agropecuarias y obras de dragado, entre otras. Por ello, la Educación Ambiental es necesaria para ayudar en la concientización de la problemática que enfrentan los manglares en la actualidad.

Desde siempre la especie humana ha interaccionado con el medio y lo ha modificado, creando problemáticas crecientes. Sin embargo, lo que hace especialmente preocupante la situación actual es la aceleración de los cambios, su carácter masivo y la universalidad de sus consecuencias. En el ámbito internacional, la Organización de las Naciones Unidas, a través de sus organismos (UNESCO y PNUMA fundamentalmente) ha sido la principal impulsora de estudios y programas relativos a la Educación Ambiental. Uno de sus propósitos fundamentales es lograr que tanto los individuos como las colectividades comprendan la naturaleza compleja del medio ambiente (resultante de la interacción de sus diferentes aspectos: físicos, biológicos, sociales, culturales y económicos) y que adquieran los conocimientos, los valores y las habilidades prácticas para participar responsable y eficazmente en la prevención y solución de los problemas ambientales, incluso en la gestión de la calidad del ambiente.

En 1992, en la Cumbre de la Tierra de Río de Janeiro (ONU, 1992), se hizo evidente la magnitud de los problemas ambientales globales, cuestión que indica aún hoy la importancia que tiene su tratamiento desde la Educación Ambiental, área de atención priorizada dentro de la currícula escolar actual en los diferentes niveles de enseñanza, incluyendo la educación no formal.

La Educación Ambiental en México ha tomado relevancia desde 1992, con las conclusiones del Congreso Iberoamericano de Educación Ambiental cele- 
brado en Guadalajara, donde se estableció que la Educación Ambiental es eminentemente política y un instrumento esencial para alcanzar una sociedad sustentable en lo ambiental y justa en lo social; no se refiere únicamente a la cuestión ecológica, además tiene que incorporar las múltiples dimensiones de la realidad. La Educación Ambiental debe fomentar la participación social y la organización comunitaria, tendientes a las transformaciones globales que garanticen una calidad de vida y una democracia plena que procure el autodesarrollo de las personas (Carvalho, 1998).

Smith-Sebasto (1997) define a la Educación Ambiental como un proceso que incluye un esfuerzo planificado para comunicar información y suministrar instrucción basado en datos científicos, así como en el sentimiento público dominante diseñado para apoyar el desarrollo de actitudes, opiniones y creencias que apoyen a su vez la adopción sostenida de conductas que guían a los individuos y grupos para que alcancen un nivel mejor de vida, para que fabriquen productos, compren bienes materiales y se desarrollen tecnológicamente, etc., de manera que minimicen la degradación del paisaje, la contaminación del aire, del agua o del suelo y las amenazas a la supervivencia de especies de plantas y animales.

La Educación Ambiental debe enseñar cómo continuar con el desarrollo al mismo tiempo que se protejen y se conservan los sistemas de soporte vital del planeta. La cultura para la conservación y rehabilitación de los bosques de manglar requiere de una mayor atención. La educación es un agente fortalecedor integral que promueve el conocimiento de los problemas del medio natural y social y los vincula sólidamente con sus causas. A través de la educación se puede enseñar a los habitantes a hacer un aprovechamiento racional del ecosistema.

\section{OBJETIVOS}

Crear conciencia en los niños de nivel primaria sobre la importancia ecológica y económica del cuidado y preservación de los recursos del manglar en beneficio de las comunidades mediante la impartición de pláticas de Educación Ambiental apoyadas con la exposición de videos, juegos y otras actividades escolares. De esta manera sería posible asegurar el éxito del proyecto Restauración de áreas de manglar impactadas por dragados en el sistema lagunar de Chantuto, el cual se está llevando a cabo con el apoyo de las comunidades de Santa Isabel y Juan Escutia, aledañas a los sitios en recuperación, para hacer uso racional de los recursos del manglar, promoviendo el cambio de valores y actitudes como una forma de mejorar la calidad de vida, abordando esta tarea educativa desde lo preventivo.

\section{METODOLOGÍA}

\section{Área de estudio}

Las comunidades Juan Escutia, y Santa Isabel forman parte de la Reserva de la Biosfera La Encrucijada en el Municipio Mapastepec en el estado de Chiapas, México, en la Planicie Costera del Pacífico, como se observa en la figura 1. El clima es de tipo $A m(w)$ cálidohúmedo, con abundantes lluvias en verano. Estas comunidades se encuentran asentadas en la parte NW del sistema lagunar de Chantuto (Fig. 1). La población está compuesta predominantemente por campesinos y pescadores, los cuales se establecieron entre 1986 y 1987, en la actualidad constituyen una 


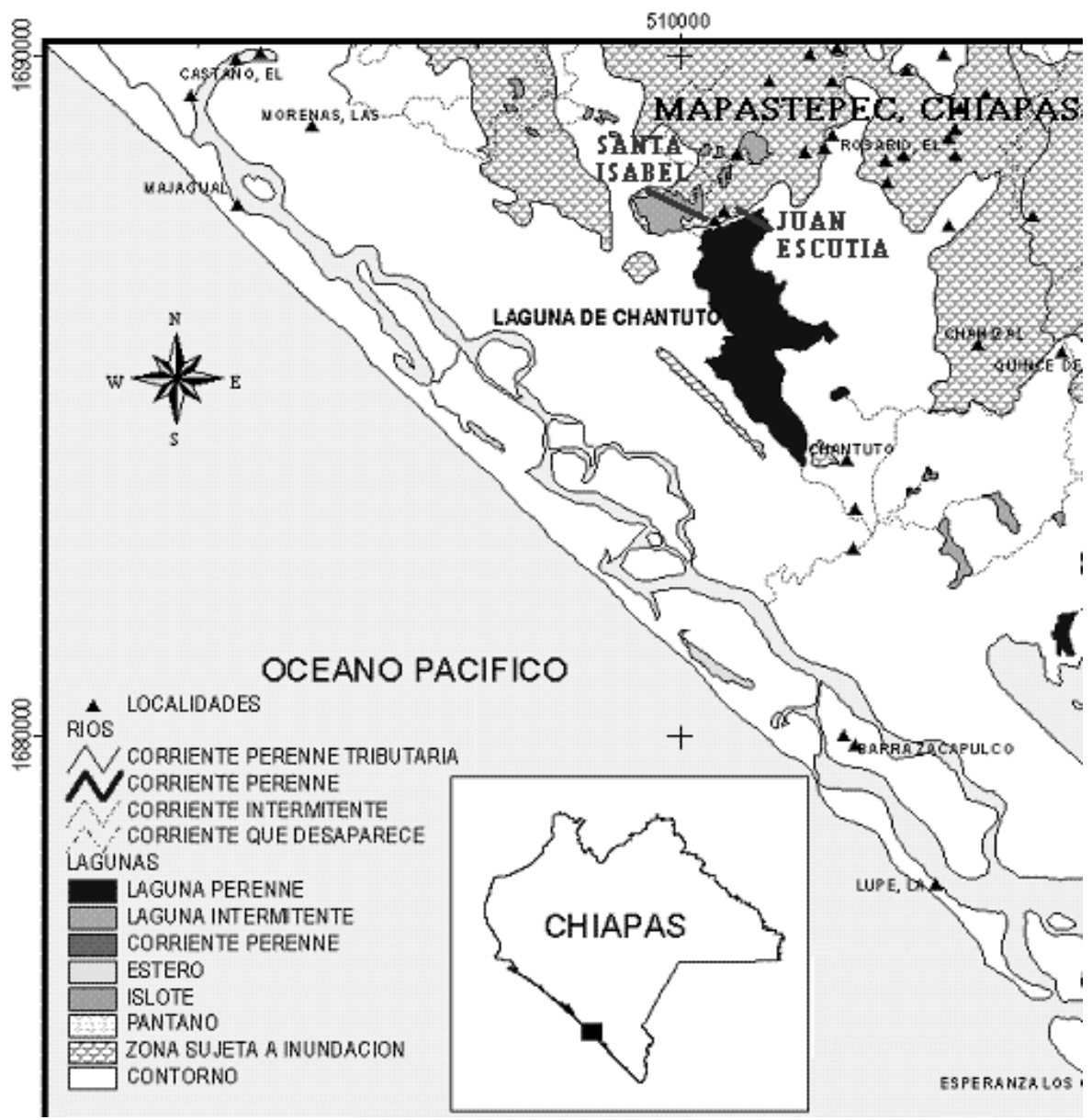

Figura 1. Localización de las comunidades Santa Isabel y Juan Escutia, municipio de Mapastepec, Chiapas México.

población de aproximadamente 680 personas.

\section{Métodos}

Las pláticas de Educación Ambiental se iniciaron el 7 de marzo del 2003 en la Escuela Primaria Rural Federal Juan Escutia. Esta escuela está constituida por un salón de clases al cual asisten 30 niños, distribuidos desde primero a sexto grado, a cargo de un maestro que proviene de un sitio externo a la comunidad. Las pláticas se realizaron cada 8 días, con una duración de aproximadamente 50 minutos, cada sesión constó de una parte teórica de 30 minutos y una práctica de $20 \mathrm{~min}$; esta última se realizó en el jardín de la escuela. El maestro de la escuela ha colaborado en todo momento, dando todas las facilidades para la realización de estas pláticas. 
A los niños, inicialmente se les muestra un panorama de cada tema a tratar, con una breve introducción acerca de la Educación Ambiental y su relación con las materias que cursan y con el medio que les rodea. En todo momento el maestro es un facilitador, que además de enseñar, fomenta la discusión entre los niños. Se utilizan diferentes apoyos didácticos que van desde láminas, acetatos, videos, hasta presentaciones en computadora, adicionalmente se realizan exposiciones y juegos variados. En cada sesión participan hasta cuatro niños exponiendo un tema, incluso analizando la importancia de éste para la comunidad. Para facilitar las pláticas se les proporciona el material a utilizar como lápices de colores, cartulinas, hojas de papel, rotafolio, pegamento tijeras, plastilina, etc. Cada niño por su parte lleva lápices de colores y pluma para la clase (Contreras, 2003).

Las pláticas se interrumpieron a inicios del mes de julio al fin del ciclo escolar y se reiniciaron en septiembre de 2003. Con el inicio del ciclo escolar se incorporó la Escuela Primaria Federal Francisco Villa de la comunidad Santa Isabel. Esta escuela está a cargo de un maestro y está constituida por dos salones de clases, donde asisten un total de 27 niños, distribuidos en dos grupos: los que saben leer y escribir y los que están en proceso de aprender. Se está trabajando con el grupo de niños que saben leer y escribir (16 niños) debido a que ellos pueden comprender mejor las platicas y realizar con mayor facilidad las actividades.

La temática de las pláticas de Educación Ambiental:

El tema El manglar, sus beneficios, deterioro, reforestación y conservación, fue uno de los más extensos, se le dió mayor consideración que a los demás, con apoyo de fotografías, láminas y videos; se mostraron a los niños los problemas que enfrentan, entre ellos el impacto del hombre en su manejo y se plantearon algunas opciones para su conservación.

Con el fin de reforzar las actividades de Educación Ambiental, el proyecto donó 160 plantas de una especie de mangle a la escuela Juan Escutia, para realizar una barrera de árboles alrededor de la escuela y del campo de fútbol, se enseñó a los niños y al profesor la manera de transplantarlos, se les proporcionó el material necesario para la siembra (cuchillos, cubetas y palas) y se les mostró como cuidarlos; los alumnos se comprometieron a regar de manera constante las plantas. Como parte de la vinculación del proyecto con la escuela Juan Escutia se donaron dos cubetas de pintura vinílica para pintar la escuela, esta actividad fue realizada por los padres de los niños. Así mismo, para fortalecer la unión entre los alumnos y padres de familia de las dos escuelas se realizó un encuentro de fútbol entre los niños. Como actividad extraescolar se celebró el día de los Reyes Magos con la tradicional rosca y una serie de juegos con los niños y en la cual participaron los padres de familia.

Una de las actividades adicionales de las pláticas de Educación Ambiental fue elaborar material educativo como folletos y trípticos para las comunidades costeras, como una alternativa para la conservación del manglar.

Con el fin de conocer el impacto de las pláticas de Educación Ambiental en los niños, se les aplicó un cuestionario con los siguientes reactivos:

1. Para ti ¿qué es Educación Ambiental?

2. ¿Qué es la contaminación?

3. ¿Cuáles son las consecuencias de la contaminación sobre la salud de las personas?

4. ¿Sabes qué es la materia orgánica y 
la inorgánica?

5. Menciona cuatro ejemplos de contaminantes.

6. ¿Qué hacen con la basura que se produce en tu casa?

7. ¿Cómo crees que se solucionaría el problema de exceso de basura?

8. Enumera 10 usos del agua en tu comunidad

9. Escribe cinco recomendaciones para cuidar el agua en tu casa.

10. ¿Sabes por qué cada día disminuye la pesca de camarón en la laguna?

11. ¿Menciona cuál es la función de los árboles en la naturaleza?

12. ¿En qué utilizamos la madera de mangle en nuestra comunidad?

13. ¿Sabes qué pasaría si se acaban los manglares?

14. ¿Qué podemos hacer para que no se corte y contaminen el manglar y la laguna?

15. Anota la importancia que tienen los animales que viven en el manglar.

16. ¿Por qué crees que haya animales en peligro de extinción?

17. Realiza e ilumina un dibujo donde describas como son tu comunidad y la laguna.

Para conocer la situación actual de las comunidades Santa Isabel y Juan Escutia en el manejo del manglar y los recursos naturales se realizó un estudio socioeconómico de manera simultánea con las pláticas de Educación Ambiental. Se realizaron encuestas a 54 familias de las dos comunidades. Los cuestionarios exploran datos personales, familiares y laborales del entrevistado, de la vivienda y del uso de los recursos naturales, entre otros.

\section{RESULTADOS}

Mediante las pláticas de Educación Ambiental y los resultados del cuestionario aplicado a los niños al finalizar el ciclo escolar, se han obtenido los siguientes resultados:

1. Comprenden cual es la función de la Educación Ambiental.

2. Conocen la problemática de la contaminación y sus consecuencias en Laguna de Chantuto.

3. Saben que deben evitar tirar basura y matar animales, aunque poco lo llevan a cabo.

4. Conocen algunas prácticas de higiene en el manejo de los alimentos.

5. Han participado en la práctica de ahorro del agua.

6. Fácilmente enumeran los usos de la madera de mangle.

7. Mencionan algunas prácticas adecuadas en el manejo y extracción racional de los recursos del manglar.

8. Saben en qué consiste y cuáles son los beneficios del proyecto de restauración en esta laguna.

Estos resultados preliminares indican la apropiación de algunos conocimientos nuevos por los niños sobre la conservación del medio ambiente, lo cual habla de un cambio de valores y aptitudes en el uso y manejo de los recursos de su comunidad. Manifiestan algunas ideas sobre posibles soluciones a la dramática reducción de la pesquería, sobre los beneficios del trabajo en equipo para el cuidado y embellecimiento del medio en el que viven, como el ejercicio de la siembra de plantas alrededor de la escuela Juan Escutia y el cuidado posterior de ellas. Otros ejemplos son la elaboración de letreros: No tirar Basura, Cuidemos a los animales, Deja vivir a las tortugas, No comas y vendas animales silvestres, Cuida el agua, etc. De igual manera se ha generado un ambiente sano de trabajo entre las cooperativas de pescadores, las escuelas, así como entre las dos comunidades y el equipo de trabajo de restauración. 
Con la información obtenida del estudio socioeconómico y del uso de recursos naturales aplicada a los adultos; asi como los talleres, se pudo saber que la mayoria de los habitantes de las comunidades no cuentan con los conocimientos minimos necesarios para el cuidado de su entorno, por lo cual las platicas de Educación Ambiental impartidas a los niños ofrecen opciones para educar a los adultos acerca de la soluciones de los principales problemas que les afectan.

Dentro de los resultados obtenidos están:

El $78 \%$ de los habitantes de estas comunidades no saben que habitan dentro de un área de reserva (Fig. 2); el $73 \%$ de los jefes de familia se dedican a la pesca por lo que la mayoria de las familias viven de esta actividad y debido a la disminución de la pesquería en algunos meses del año se tienen que dedicar al campo para poder sobrevivir (Fig. 3). La mayoria de los habitantes de las comunidades utilizan un fogón para cocinar sus alimentos y $73 \%$ utiliza el mangle como len̂a (Fig. 4); mientras que $66 \%$ de las viviendas están construidas con madera de mangle (Fig. 5); en estas comunidades no hay un buen manejo de la basura (Fig. 6). El $96 \%$ de los habitantes que utilizan el mangle como len̂a, lo cortan en las inmediaciones de las comunidades, por lo que estos sitios son los más vulnerables.

\section{Discusión}

Para lograr una conducta ambiental positiva que faworezca la conservación del medio, las estrategias de Educación Ambiental deben involucrar todos los aspectos formativos de los niflos (inteligencia, emociones y experiencias), los cuales son factores que influyen en la predisposición hacia el compromiso y la acción con el resto de la comunidad en el beneficio común. Todo el proceso educativo debe desembocar en la acción positiva sobre el entomo de mejora, de sensibilización, de solución de problemas, incluso de prevención, etc. Las actividades de Educación Ambiental deben incluir herramientas pedagógicas y dinámicas, grupales de juegos y de motivación para crear un ambiente agradable y que se logre fácilmente la adquisición de conocimientos para la conservación del medio ambiente. Para realizar estas actividades el docente debe de tener una gran predisposición de servicio y una

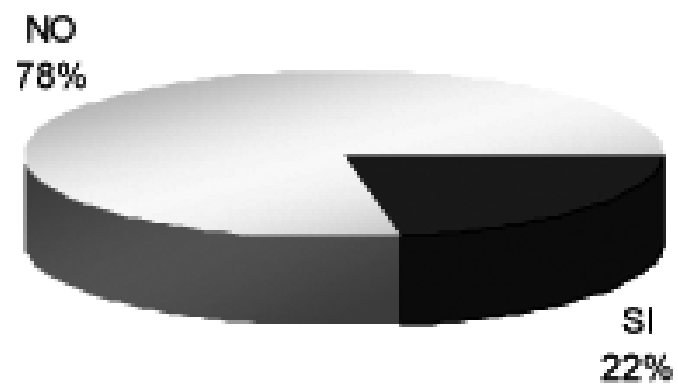

Figura 2. Conocimiento de los habitantes acerca del área donde viven es una reserva de la Biosfera. 


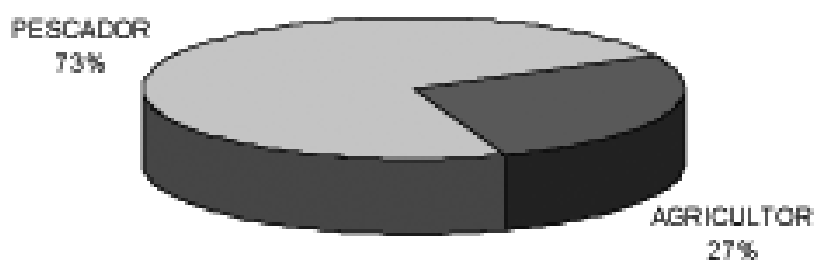

Figura 3. Ocupación de los jefes de hogar.

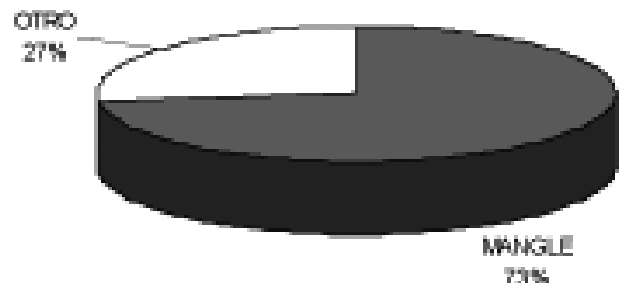

Figura 4. Material utilizado como leña.

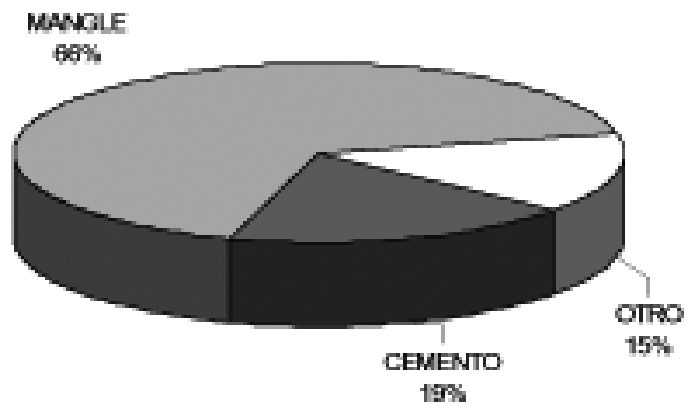

Figura 5. Material utilizado en la construcción de las viviendas.

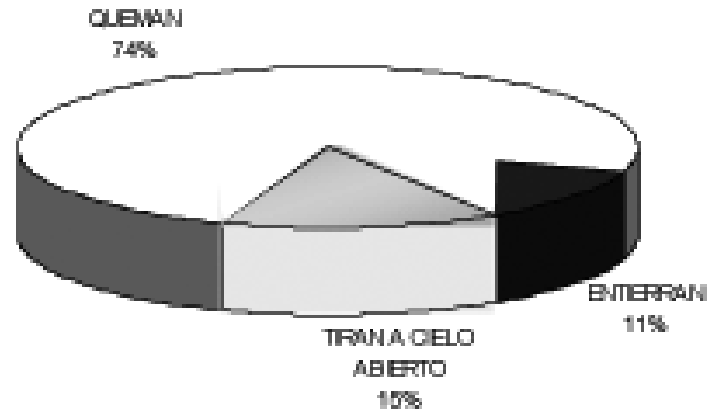

Figura 6. Manejo de la basura. 
buena dosis de altruismo, debido a la localización geográfica y situación social de las comunidades, además de contar con los recursos económicos para poder realizarlas y brindar el material necesario a los participantes.

La Educación Ambiental es una alternativa para la conservación de la zona costera en su conjunto, por lo que se debe utilizar con fines preventivos para evitar el deterioro y agotamiento de los recursos de nuestras costas. Cada uno de nosotros debe de practicarla en todo momento con el fin de formar conciencia en los niños de las normas y valores que se tienen que adoptar sobre el cuidado de nuestro entorno, incluso la ética del comportamiento humano del uso y desuso de los recursos comunes de este planeta. Citando a Houstoun (1994): La crisis ambiental no está tanto en el crecimiento de la población, el desarrollo industrial, ni en el sistema económico y político, sino en las actitudes y valores que motivan las decisiones humanas.

La Educación Ambiental, debe desarrollar el conocimiento necesario para que el individuo pueda concebir las consecuencias de sus actos y para que pueda reconocer los sacrificios que la elección de los bienes ambientales implica para la sociedad. No se trata sólo de difusión de conocimientos existentes. Es fundamental, para lograr el cambio necesario, que la Educación Ambiental no sea transmisora de la cultura sino transformadora de la misma y esto lo deben entender aquellos quienes divulgan la Educación Ambiental, de lo contrario, el esfuerzo será en vano.

\section{CONCLUSIONES}

Las pláticas de Educación Ambiental han sido un buen apoyo durante el proyecto de restauración, primero porque ha permitido el acercamiento del equipo de trabajo con las comunidades que laboran en la restauración de áreas de manglar afectadas por obras de dragado $y$, segundo porque ha resaltado la importancia del manglar, ha promovido su uso racional y su conservación así como el cuidado del medio ambiente; con esto, las comunidades están tomando conciencia para realizar el manejo adecuado y el cuidado de las áreas de manglar y se está logrando la transformación de personas no sensibilizadas a personas informadas, sensibilizadas y dispuestas a participar en la resolución de los problemas ambientales. Con estas actividades se transmitió información a comunidades que contaban con escaso conocimiento del cuidado del medio ambiente y de las posibles alternativas de manejo que permitirá detectar, controlar y minimizar los efectos de las actividades humanas, con la finalidad de que las acciones que se efectúen en el aprovechamiento del manglar sean las más adecuadas para su conservación.

\section{REFERENCIAS}

Carvalho, I. 1998. Las transformaciones de la cultura y el debate ecológico: desafíos políticos para la Educación Ambiental Formación Ambiental. Red de Formación Ambiental para América Latina y el Caribe. PNUMA. Vol. 10 № 22. México. FebreroJunio. p. 15.

Contreras, D. Mendoza. A. 2003. Educación Ambiental. Ed. Esfinge. México.

Houstoun, H. 1994. Proyectos verdes. Manual de actividades participativas para la acción ambiental. Biblioteca Ecología. Ed. Planeta.

ONU (Organización de las naciones Unidas) 1992. Declaración de Río sobre el medio ambiente y el desarrollo y programa 21 (1993). In: Informe de la Conferencia de las 
Naciones Unidas sobre el Medio Ambiente y el Desarrollo. Río de Janeiro. 3 a 14 de junio de 1992 . Vol. I. Resoluciones aprobadas por la Conferencia de Naciones Unidas. Nueva York.

Ruiz-Durá, F. 1985. Recursos pesqueros de las costas de México. Ed. Limusa. México.

Smith-Sebasto. 1997. Environmental Issues Information Sheet El-2. Illinois University. EUA.

Tovilla H., C. 1998. Ecología de los bosques de manglar y algunos aspectos socioeconómicos de la zona costera de Barra de Tecoanapa, Guerrero, México. Tesis Doctoral. Facultad de Ciencias. Universidad Nacional Autónoma de México. 368p.

UNESCO-PNUMA. 1994. Publicaciones del programa internacional de educación ambiental. Los libros de la Catarata. Bilbao.

Wuest, T. 1992. Ecología y educación: elementos para el análisis de la dimensión ambiental en el currículum escolar. CESU/UNAM. México. $\diamond$ 